\title{
Teaching Agent Model Construction Based on Web Cooperative Learning System
}

\author{
Li Yaolin \\ Department of Information Technology \\ JiangMen Polytechnic \\ Jiangmen, China \\ lilin1566@126.com
}

\author{
Yanhua Zhong \\ Department of Information Technology \\ JiangMen Polytechnic \\ Jiangmen, China \\ zhflowers@163.com
}

\author{
Zhang Zongfu \\ Department of Information Technology \\ JiangMen Polytechnic \\ Jiangmen, China \\ jmptzhang@126.com
}

\begin{abstract}
The research tries to solve the problems related to teaching agent technology in cooperative learning system. Aiming at the fact of present learning system being unable to realize real-time intelligent teaching for students, a creative idea of teaching agent mode based on web cooperative learning system is put forward after studying the technology of student agents' reasoning algorithm, learning path agent, and teacher agents. This mode may help to realize self-adaption teaching in cooperation center on digital campus. Besides, it provides new ideas or methods of internal demands for establishing web cooperative learning center. Fact shows that teaching agent can improve teaching effect and quality through individualizing the teaching materials and attracting the students.
\end{abstract}

Keywords- teaching agent; cooperative learning; student agent; model

\section{INTRODUCTION}

Digital learning has become an important field in research of education technology, with the rapid development of wireless network technology, intelligent mobile phone communication technology, intelligent agent technology, as well as the rise of micro mobile learning that is to say students can get an access into collaborative learning system by computer, laptop, intelligent mobile phone and other devices. Once some kind of simple technique could help teachers making network course and teaching management, which could finish amount of work instead of teachers while under their will, teachers would be free from the time-consuming manual monitoring jobs. Kinds of new technology are used to improve the deficiencies of remote teaching based on web, such as data warehouse, data mining, CSCW, artificial intelligence, middleware and agents. Among them, the agent technique is especially suitable for the remote digital learning. Intelligent Agent technology theory and technology research is expanded from distributed artificial intelligence (DAI) field. Pedagogical agent is one of the intelligent agents, which must be able to understand teaching instructions and apply them to an object or action. The dialogue between teacher (the agent controller) and object should also be supported by the agent, initiated either by teacher or agent. What's more, it's necessary for agent to have ability to learn teaching instructions and carry them on different situations ${ }^{[1]}$.
A kind of teaching agent model is put forward for realizing self-adapted teaching in cooperative center of digital campus. It would be benefit to students during their online learning process by exchanging and sharing knowledge among various teaching agents. As a result, the following could be achieved: students learning what they desire, teachers assigning courses according to students' aptitude, adding more interesting and humanizing content in teaching, improving the teaching effect, increasing the teaching quality.

II. DESIGN AND IMPLEMENTATION OF TEACHING AGENT MODEL BASED ON WEB COLLABORATIVE LEARNING SYSTEM

The teaching agent model based on web collaborative learning system is formatted by student group, teacher group, teaching agent, database group, as shown in Figure1. The student group refers to all online learning students. The teacher group refers to all the teachers teaching online. The teaching agent includes student agent, teacher agent and collaborative agent. Student agent can assist student's learning and automatically record their learning path. It would provide the cognitive level according to student's related test result and offer knowledge-point search and question answering system to solve student's questions. The teacher agent can analyze and advice on problems to student, and answer the unresolved issues in self-answering system, based on the student's cognitive level reasoned by student agent and investigation of student information and interesting. The collaborative learning agent is helpful to teacher agent get the situation of all student agents and facilitates the communicating and exchanging learning experiences to solve problems among all student agents. The database group includes knowledge base, assignment base, question base, evaluation base, and information base. The whole storage of knowledge-point, learning path, student information, cognitive level and etc in database group could provide necessary data information to student agent for analyzing their studying and teacher agent for developing teaching strategies.

The first time student logging into collaborative learning system needs to be registered. The system will prompt him to choose the type of proxy service, select the teaching agent or self-learning. If student chose to be controlled by the teaching agent, he had to accept the investigation of student information and learning interest, finish the basic 
information and save in the information base. After entering the learning system, it would transfer corresponding content to their learning to student. And student agent would record student's access to knowledge-point and save in student information database. Cognitive level assessment agency will give student questions of different level and difficulty in order to test and evaluate their level of awareness. The simple problems encountered during student learning process would be solved by knowledge-point and selfanswering system of student agent, which could bring up the answers to learners. And the questions beyond the scope of self-answering system, student agent will request a connection to teacher agent. Then the teacher agent will analysis and answer them directly. At the same time, teacher agent can make personalized learning recommendations to students according to their information and interest trend, and find their learning problems in order to put forward solutions. Student agent, teacher agent and the rest various agents could request simultaneous interactive communication and collaborative learning between each other to the collaborative agent. But for more complex ones, which both student agent and teacher agent can not give answers to, must be unified planned and consulted by collaborative learning agent.

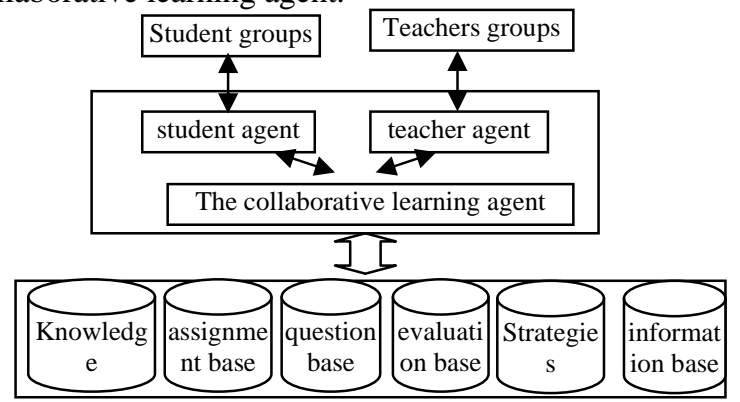

Figure 1. The teaching agent model based on web collaborative learning system

\section{STUDENT AGENT}

Student agent completes students' learning recording, learning path record in other words, as well as evaluates the cognitive level of each student through tests. It also provides knowledge-point search function. At the same time, students can pose questions to teacher agent by using self-answering system.

Why Fuzzy logic reasoning method is adopted in the reasoning process of student agent mainly because of the following consideration. The fuzzy set is more suitable on behalf of the interaction behavior between user and system. Because the process of student's learning is constant and transforming all the time, the system only can adopt approximate reasoning. So the fuzzy logic is more suitable for the reasoning. It provides direct and low complexity reasoning, which is easier than other methods like Bayesian network. Although the results of using fuzzy theory are less accurate than those of Bayesian network, it provides a much similar probability value.

\section{A. the representation on Students' cognitive level}

Cognitive level indicates the degrees of knowledge possessed by students. It's a gradual process for student to master knowledge. And the understanding of system for student's degree is with a lot of uncertainties. For examples, when student browses the content of some knowledge, the agent will think his knowledge level of this point would increase, but this speculation has great uncertainty; while the student has done the exercise of the knowledge point, the agent evaluates his cognitive level through his exercise; however, the uncertainty of this evaluation is not great. The cognitive level of student is dynamic with great uncertainty. As a result, fuzzy sets are used to represent student's cognitive levels. The student's cognitive level is divided into 4 grades. Each grade of membership is expressed as $\mu_{k}(i), i=1 \sim 4$

, using $\mathrm{K}$ to represent the student's cognitive level fuzzy sets, $K$ to be expressed as $k=\sum_{i=1}^{4} \frac{\mu_{k}(i)}{i}, 0 \leq \mu_{k} \leq 1, \sum_{i=1}^{4} \mu_{k}(i) \leq 1$

For an example: The four grades of membership degree of a student's cognitive level is $\{0.2,0.1,0.2,0.4\}$.His cognitive level is expressed as $\mathrm{K}=0.2 / 1+0.1 / 2+0.2 / 3+0.4 / 4$, Here the "+" symbol is a Boolean connectors "and." According to K, the expression, level 4 is the highest degree of membership which can estimate the level of knowledge the student most likely to reach the fourth grade. ${ }^{[2]}$.

\section{B. The method of changing fuzzy set membership for student's cognition level}

Student's cognitive process is a dynamic process, so the evaluation of their level of knowledge should be changed as his cognitive level changing. So it requires the system can changing fuzzy set membership for student's cognition level dynamically. Here is the way to change the fuzzy membership, which is an improvement to the method adopted by Sherlock II system.

Let $\mathrm{m}$ is the level of question, the higher level meaning the greater difficulty; i refers to the student's cognitive level

of knowledge point; $q_{i}$ stands for the degree of membership of $\mathrm{i}$; $\mathrm{c}$ is a constant, taking $\mathrm{c}=0.5$.

$$
\begin{array}{ll}
i<m: & q_{i}=q_{i}-q_{i}{ }^{c}+q_{i-1}{ }^{c} \\
i=m: & q_{i}=q_{i}+q_{i-1}{ }^{c}+q_{i}{ }^{c} \\
i>m: & q_{i}=q_{i}-q_{i}{ }^{c}+q_{i+1}{ }^{c}
\end{array}
$$

Basic principle is that when users have done the test of a level in the right way, the low levels should be used the following rules and levels above should be used the drop rules. When the user can not pass the tests of a level, that is to say he does not meet the level requirement, his level of 
awareness world not be changed. Examples are shown in Table 1.

TABLE I. THE TESTS OF A LEVEL

\begin{tabular}{|c|c|c|}
\hline Initiate & 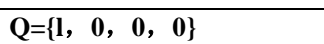 & $c=0.5$ \\
\hline 1 & $\mathrm{Q}=\left\{\begin{array}{llll}0.5, & 0.5, & 0,0\end{array}\right\}$ & $\begin{array}{l}\begin{array}{l}\text { Level } 2 \text { of tests is } \\
\text { passed }\end{array} \\
\end{array}$ \\
\hline 2 & $\mathrm{Q}=\left\{\begin{array}{llll}0.25, & 0.75, & 0, & 0\end{array}\right\}$ & $\begin{array}{l}\text { Level } 2 \text { of tests is } \\
\text { passed }\end{array}$ \\
\hline 3 & $\mathrm{Q}=\left\{\begin{array}{llll}0.125, & 0.5, & 0.375, & 0\end{array}\right\}$ & $\begin{array}{l}\text { Level } 3 \text { of tests is } \\
\text { passed }\end{array}$ \\
\hline 4 & $\mathrm{Q}=\left\{\begin{array}{llll}0.125, & 0.5, & 0.375, & 0\end{array}\right\}$ & Level 3 of tests is failed \\
\hline 5 & $\mathrm{Q}=\left(\begin{array}{llll}0.125, & 0.5, & 0.375, & 0\end{array}\right)$ & Level 3 of tests is failed \\
\hline 6 & $\begin{array}{l}Q=\{0.0625,0.75,0.1875, \\
0\}\end{array}$ & $\begin{array}{l}\text { Level } 2 \text { of tests is } \\
\text { passed }\end{array}$ \\
\hline
\end{tabular}

From this table we can determine the awareness of the student belong to the second grade. Although he chooses the right answer when he does the No.3 question, his answer to the No.4 question of the same level is wrong. We can believe that he could guess the right answer for the former or neglect the latter. The same level of No.5 is wrong again, we can infer that he does not achieve this level and lower his level. As a result of this method and topics of algorithm, we can exclude the interference of some information such as: guess or negligence. It's better of the classification of user's understanding. In summary, the combination of improved the level of rules and tests, is more similar to the actual situation

\section{C. the self-adaptive of random problem algorithm}

To complete the self-adaptive, we based on the cognitive level agency to design a random problem algorithm. Algorithm is as follows: Let i denote the level of test, test includes four levels of primary, familiar, master and apply, $\mathrm{d}$ indicates the difficulty of the test (d values of $l \sim 5$, the order is easy - difficult), S [i ] [d] records level of difficulty for the number of test answered correctly.

a. Initialize $\mathrm{i}=2, \mathrm{~d}=3$, the initial array $\mathrm{S}=0$;

b. If $1 \leq i \leq 4$ and $1 \leq d \leq 5$, then taking i-level, $\mathrm{d}$ level difficulty of the tests;

If $2 \leq i \leq 4$ and $d<1$, then $i=i-1, d=5$;

If $1 \leq i \leq 3$ and $d>5$, then $i=i+1, d=2$;

If $i<1$, then go to d;

If $i>4$, then go to d; b;

c. If the tests answered correctly, the difficulty of $d$ up to

If the tests answered incorrectly, the difficulty of $d$ down to b;

d. Refresh the page again the topic.

Using this algorithm with the cognitive level agent reasoning, the self-test can be adaptive test.

\section{Learning path agent}

Learning path agent can dynamically record the student's current knowledge of access points webpage, which can be displayed the accessed situation to the students at any time. Its main purpose is to record the students in the learning network courses, what has been learned or what not. According to these circumstances, agents may prompt students for further learning contents. When students visit the page of a knowledge point, the path agent will be recorded automatically the page address in the address bar into learning log table by the program. At the same time learners ' login ID and time information will be recorded into database. After learning this chapter, it will display what will be learned based on learning point recording. Advising the users on how to enter the following learning content and reach the system requirements.

Recording the path of action learning is completed when the page loads, each page of knowledge in the Unload event point is triggered when the call to the insert function a JScript script file, hisinsert.js. onLoad="MM”_calljs('hisinsert/agentjsp/hispathindb.jsp)')

This function will access the webpage to get the current URL, which you can get the file name strurl... hisinsert.js script code is as follows:

Function hisinsert(theURL)

\{

theURL $=$ theURL + , ?struri $=$,

+document.location.href;

window.location $=$ theURL:

\}

Function by calling hisinsert parameter theUR our work gives'.../../ agentjsp / hispathindb.jsp) 'value, which reached the purpose of direct access to hisPathindb.jsp file, the JSP file to the database is completed insert the path to get strurl knowledge point information, so as to achieve the purpose of recording knowledge point information. In connection with the database in the process, JDBC data access ways are used.

\section{E. Intelligent agent}

Students can present their problems to the self-answering system during the network learning process, which can provide answers to students directly. When the problems beyond the scope of self-answering system, it can get solving methods from the teacher agent. Self-answering system provides an important direct link way for student agent and teacher agent. At present, the mechanism of system answers is simple string matching, and its ability of reasoning and correctly answering is very low. Therefore, to improve the answering ability of self-answering system, a mechanism for questions analysis is necessary. Students could combine their habits with the thinking ways to ask questions, so the system can help them to correctly express their problems.

\section{TEACHERS AGENT AND SYNERGISTIC AGENT MODULE}

Teacher agent can complete the analyst of cognitive level and interest in learning. At the same time it could be concerned about the learning of all students. Students will be grouped with the same level or formed study groups and collaborative learning. Finally, each of students can improve learning efficiency. Teacher agents can determine students' 
learning progress, basing on the situation analysis, which can help arrange the learning content for individual. Through analysis-answer system, teacher will be connecting with the student's proxy answering system to help students solve questions.

Collaborative learning agent is in charge of the communication between student agent and teacher agent. Whether student agent or teacher agent is available to share the information exchanging and solve difficult problems together. Co-learning agent is mainly used dynamic methods such as electronic whiteboard, messaging delivery, online discussion and message board. Collaborative learning agent mostly supports collaborative and partners-learning ways. It provides with the following mechanism to coordinate the student agent and teacher agent, particularly for the collaborative learning among student agent.

\section{DATABASE GROUP}

Learning content implies the front module is included in the next module; it can be easily set when and where can entry the knowledge point between the adjoining points. At the same time, the similar knowledge points have correlation, and chapters knowledge points have the relation of apparently contains. The following example shows setting up mode of the relationship between the coefficients and the premise:

Definition 1 Knowledge module prerequisite relation.

Set $\varphi_{i, j}$ ( Representation of knowledge module ) is $D_{i}$ and $D_{j}$ Prerequisite relation, Then $\varphi_{i, j}$ Can be expressed as:

$$
\varphi_{i, j}=\left[\begin{array}{cr}
1 & D_{i}^{i s} D_{j} \text { prerequisite } \\
0 & \text { other relations } \\
-1 & D_{i}^{i s} D_{j}^{\text {follow - up }}
\end{array}\right]
$$

Thus, the inputs of knowledge modules are set up below the anti-symmetric matrix:

$$
\varphi_{n, n}=\left[\begin{array}{cccc}
\varphi_{1,1} & \varphi_{1,2} & \cdots & \varphi_{1, n} \\
\varphi_{2,1} & \varphi_{2,2} & \cdots & \varphi_{2, n} \\
\cdots & \cdots & \cdots & \cdots \\
\varphi_{n, 1} & \varphi_{n, 2} & \cdots & \varphi_{n, n}
\end{array}\right]
$$

Here, $\varphi_{i, j}=-\varphi_{i, j}, n$ expresses the number of knowledge modules in knowledge stock ${ }^{[3]}$

\section{APPLICATION CASE}

In the "modern educational technology" network course, based on the teaching agent model of the network collaborative learning system, the student agent, teacher agent and collaborative learning agent and other modules are added into it. Beaweblogics. 0 as the application server for publishing web pages is used in the collaborative learning system of teaching agent in "Modern educational technology". In order to realize some specific function, student agent, teachers and reasoning part of the agent interface, message boards of collaborative learning agent, chat rooms and e-mail delivery, the dynamic web-design of database access part adopts JSP / DBBean technology. Through the simulation tests, the each module's function of system is basically to achieve the intended design requirements and can serve as a practical system for students, including the use of exercise testing for cognitive level, learning path recording function tests, knowledge tests point search.

\section{CONCLUSION}

By building teaching agency model based on web collaborative learning system, it provides new ideas and methods for the digital campus construction, which can achieve the basic functions of teaching agents. Yet the effect is not very satisfied. Therefore, the algorithm should be improved further and the model should be perfected. Only making good use of knowledge structure, intelligent agent technology and software technology, can we solve the personalized intelligence to guide learning and intelligent question answering, develop an effective online teaching system solution.

\section{REFERENCES}

[1] D.H. D.H., Jonathan. Thought of Technology Supporting [M]. Shanghai:ECNUP,2008: (Ch).

[2] Wang Rui .Research and Design of Pedagogica1 Agent of Cooperative Web-Learn1ng System[J]..Dalian Maritime University, Master's thesis , 2004 (02):27-35 (Ch).

[3] Katz.S.Lesgold.A.Eggan.Gordin.M,ModelingthestudentinSherlock11 .JournalofArtifieialIntelligeneeinEdueation. 2000:495-518

[4] Chen Ziyu.Research on Far-Distance Teaching System and Students Model Based on Agent [J]. Thesis for Master's Degree, Chong Qing University. 2002,5(1):46-49 (Ch).

[5] Fei Yulian; Wang Xun, Zhang Lei. Design and Development of WebTeaching System Based on AGENT[J]. Computer Application and Microsoft ,2003,(12): 19-20. (Ch). 